\title{
NUMERICAL SIMULATION OF TWISTED SOLAR CORONA
}

\section{S. PARHI ${ }^{1}$, B. P. PANDEY ${ }^{2}$, M. GOOSSENS ${ }^{3}$ and G. S. LAKHINA ${ }^{2}$ \\ 1 Communications Research Laboratory, Tokyo 184, Japan \\ 2 Indian Institute of Geomagnetism, Bombay 400 005, India \\ ${ }^{3}$ Katholieke Universiteit Leuven, Leuven, Belgium}

\section{Introduction}

The solar corona supports a variety of waves generated by convective upwelling motion in the photosphere. In order to explain the observed coronal temperature profile, resonant absorption of MHD waves by coronal plasma (Goossens et al., 1995) has been proposed as a possible candidate. The physical picture is that the footpoint motion in the photosphere constantly stirs the coronal plasma leading to the MHD wave generation which is then resonantly absorbed producing the enhanced heating of the corona. Here we consider the problem of MHD wave propagation in a twisted solar corona.

\section{Governing Equations and Boundary Conditions}

The coronal plasma in cartesian geometry obeys the usual compressible, time-dependent and resistive equations. The equations are solved in a rectangular spatial domain $D=[-5 a,+5 a] \times[-8 a,+8 a]$, using a $2.5 \mathrm{D}$ resistive MHD code. Rigid wall and free-slip boundary conditions are considered at the top and bottom of the simulation regions: $V_{y}=0$ and $\partial f / \partial y=0$ for $f=\rho, p, \mathbf{B}$, $V_{x}$, and $V_{z}$. At the remaining $(x)$ boundaries open (zero gradient) conditions for all of the variables are considered (Parhi et al., 1996; Parhi et al., 1997).

We present all our numerical results for the Lundquist number $a V_{a} / \eta=$ 2000 and plasma $\beta$ varying from $5 \times 10^{-3}$ to 0.95 . The equilibrium density $\rho_{0}(x)=\rho_{e}+\left(\rho_{i} / \rho_{e}-1\right) \rho_{e} \operatorname{sech}^{3}(|x|-a)$ for $|x|>a$ and $\rho_{0}(x)=\rho_{i}$ for $|x| \leq a$ with $\rho_{i} / \rho_{e}=4$ and $V_{a e} / V_{a i}=3 . B_{o y}(x)$ has similar structure and $B_{0 z}(x)=c x e^{-x^{2}}$, where $c$ is the twist parameter. At $x=-8 a, V_{z}$ is perturbed by $F_{z}(x, y, t)=F_{d}(x) \sin \left(\omega_{d} t\right)$, where $F_{d}(x)$ has similar structure as $\rho_{0}(x)$. The following is considered: $F_{d}=0.02 \rho_{e} V_{a e}^{2} / a, c=0.06 V_{a e} \sqrt{\mu \rho_{e}}$ and $\omega_{d}=0.5$ $V_{a e} / a$.

\section{Numerical Results}

The driver excites Alfvén wave which in turn excites the magnetosonic waves. Consequently, these waves are resonantly absorbed in the corona leading to coronal heating. The evolution of plasma through sausage or kink modes 
depends on the amount of shear in the magnetic field (the following figure at $\left.t=200 a / V_{a e}\right)$. As plasma evolves the current sheets which provide the heating at the edges are distorted and fragment into two current sheets at each edge which in turn come closer when the twist is enhanced. The twist facilitates the formation of current sheet which produces the heating. Increase in the shear of the magnetic field causes the resonance layer to shift to the middle of the coronal tube. Steep gradients in slow wave at the slab edges which are signature of resonance layer where dissipation takes place are observed. It is observed that the thickness of the Alfven resonance layer is more that that of the slow wave resonance layer. Effort is made to distinguish between slow and Alfvén wave resonance layers.

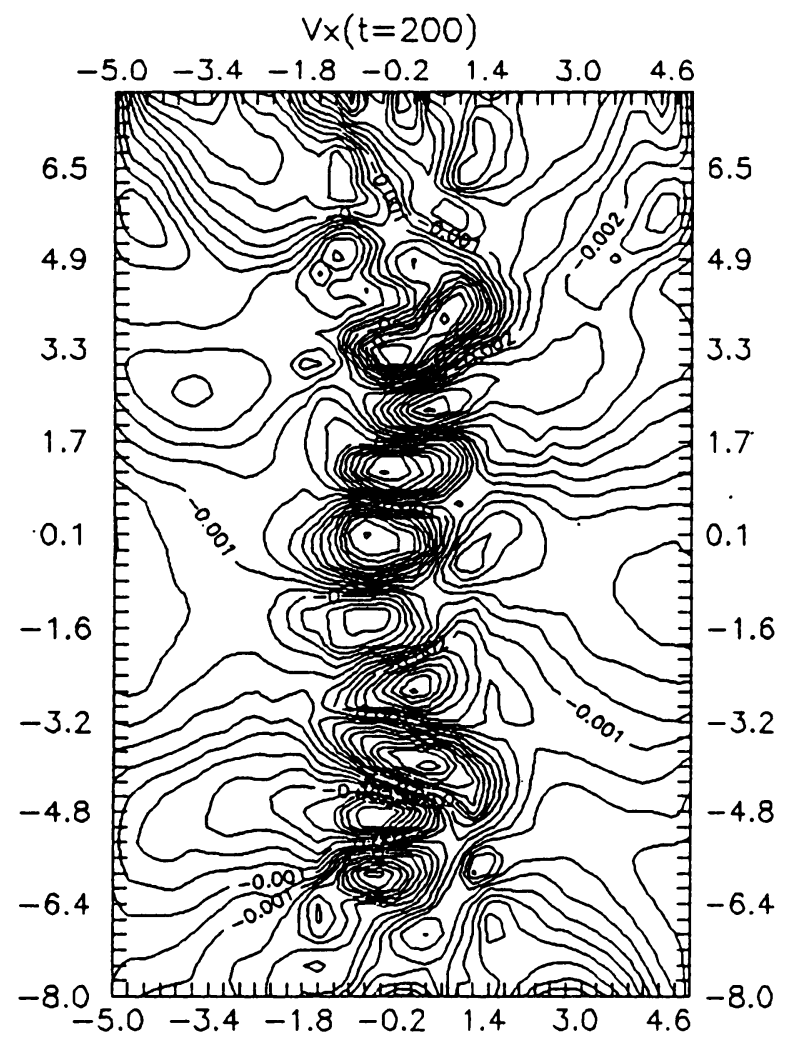

\section{References}

Goossens, M., Ruderman M. and Hollweg, J. V.: 1995, Solar Phys., 157, 75.

Parhi, S., De Bruyne P., Murawski, K., Goossens, M. and DeVore, C. R.: 1996, Solar phys. 167, 181.

Parhi, S., Pandey, B. P., Goossens, M., Lakhina, G. S. and De Bruyne P. : 1997, Astro. Space Sci., in press. 